Revue d'histoire de l'Amérique française

RAS REVUE D.HISTOIRE DE L'AMÉRIQUE FRANÇAISE

\title{
Célébrations de l'histoire et pratiques de communication publique
}

les « Fêtes de la Nouvelle-France » de Québec en 2002

\section{Josette Brun}

Volume 57, numéro 1, été 2003

L'histoire " publique » : un enjeu pour l'histoire

URI : https://id.erudit.org/iderudit/008357ar

DOI : https://doi.org/10.7202/008357ar

Aller au sommaire du numéro

Éditeur(s)

Institut d'histoire de l'Amérique française

ISSN

0035-2357 (imprimé)

1492-1383 (numérique)

Découvrir la revue

Citer cet article

Brun, J. (2003). Célébrations de l'histoire et pratiques de communication publique : les " Fêtes de la Nouvelle-France " de Québec en 2002. Revue d'histoire de l'Amérique française, 57(1), 101-115.

https://doi.org/10.7202/008357ar d'utilisation que vous pouvez consulter en ligne. 


\title{
Célébrations de l'histoire
}

\section{et pratiques}

\section{de communication publique :}

les "Fêtes de la Nouvelle-France"

de Québec en 2002

JOSETTE BRUN

Département d'information et de communication

Université Laval

\begin{abstract}
Cet article s'intéresse aux festivités à caractère historique et aux pratiques de communication publique par l'examen de la couverture journalistique de la sixième édition des Fêtes de la Nouvelle-France (FNF), tenues à Québec en $2002^{1}$. Comme les commémorations, ces manifestations récréotouristiques annuelles sont au cœur d'enjeux économiques, sociopolitiques et culturels de taille ${ }^{2}$. C'est en partie par le
\end{abstract}

1. Josette Brun, «La vulgarisation historique comme exercice de communication publique: les Fêtes de la Nouvelle-France de Québec en 2002 ", communication non publiée, Congrès de l'Association francophone pour le savoir (Rimouski, 2003). Je tiens à remercier les responsables des Fêtes de la Nouvelle-France, qui ont facilité ma cueillette de données sur les Fêtes de 2002 en me fournissant notamment le plan directeur adopté en 2000, le plan de communication, le rapport d'activités et la revue de presse des Fêtes de 2002. Je remercie aussi Patrice Groulx, qui a bien voulu commenter une première version de l'article, ainsi que les évaluateurs du manuscrit.

2. Deux articles traitent de fêtes historiques qui se sont tenues vers la fin du $\mathrm{xx}^{\mathrm{e}}$ siècle à Québec. Marie-Claude Rocher, "Tradition et création: la Ducasse de Mons et les Médiévales de Québec», dans Marie-Claude Rocher et André Ségal, dir., Le traitement du patrimoine urbain: intégration, intégralité, intégrité (Québec, Musée de la Civilisation, 1997), 189-202, Actes du Colloque Mons-Québec 1996; Richard Larue et Fabrice Montal, "Québec 1984: le silence des historiens", dans Jacques Dagneau et Sylvie Pelletier, dir., Mémoires et histoires dans les sociétés francophones, Actes du Célat, 7 (juin 1992): 49-56. Plusieurs études se sont intéressées aux commémorations au Québec et au Canada. La ville de Québec est au cœur des études de H. V. 
biais de la communication publique qu'elles prennent vie, particulièrement depuis l'essor sans précédent qu'ont connu les médias de masse ainsi que les domaines des relations publiques et de la publicité au cours des dernières décennies ${ }^{3}$. La réflexion qui suit cherche à jauger l'intérêt que portent les organisateurs des FNF et les médias à l'histoire, telle que présentée dans le cadre de cette célébration et en tant que champ d'étude scientifique, et à analyser les représentations que l'on fait des femmes en Nouvelle-France et du débat entourant leur statut, dans les quotidiens de la ville de Québec ${ }^{4}$. L'analyse de contenu porte sur le plan directeur adopté en 2000 ; le plan de communication, le programme, le dépliant et la publicité entourant les célébrations de 2002; et la revue de presse réalisée pour le compte des organisateurs après ces festivités ${ }^{5}$. Ce petit corpus, qui convient à une première exploration du sujet, sera élargi dans le cadre d'un projet plus vaste portant sur les FNF depuis 1997.

Il est entendu que la nature commerciale et touristique d'un tel événement, de même que les contraintes entourant la production journalistique, rendent difficile la transmission de connaissances historiques dans une perspective critique faisant place aux débats ayant cours dans la

Nelles, L'histoire spectacle: le cas du tricentenaire de Québec (Montréal, Boréal, 2003) et de Ronald Rudin, Founding Fathers: The Celebration of Champlain and Laval in the Streets of Québec, 1878-1908 (Toronto, University of Toronto Press, 2003). Des chercheurs en communication s'intéressent pour leur part à l'utilisation de l'histoire dans les entreprises. Voir notamment Chantal de Corte, "La célébration d'anniversaires comme activité de relations publiques: le cas du centenaire du Mouvement Desjardins", mémoire de maîtrise, Université Laval, 2002.

3. La communication publique est définie comme «l'ensemble des phénomènes de production, de traitement, de diffusion et de rétroaction de l'information qui reflète, crée et oriente les débats et les enjeux publics; la communication publique étant non seulement le fait des médias mais aussi des institutions, entreprises, mouvements et groupes qui interviennent sur la place publique», Michel Beauchamp et al., Communication publique et société, repères pour la réflexion et l'action (Boucherville, Gaëtan Morin, 1991). Lire dans le même ouvrage le survol historique de Jean de Bonville, "Le développement historique de la communication publique au Québec», 1-50.

4. Sur le rapport entre les médias et l'histoire, voir Jean-Pierre Rioux, «Histoire et journalisme. Remarques sur une rencontre», dans Marc Martin, dir., Histoire et médias : journalisme et journalistes français, 1950-1990 (Paris, Albin Michel, 1991), 192-205; Maryline Crivello-Bocca, L'écran citoyen. La révolution française vue par la télévision de 1950 au Bicentenaire (Paris, L'Harmattan, 1998); Isabelle Veyrat-Masson, Quand la télévision explore le temps. L'histoire au petit écran 1953-2000 (Paris, Fayard, 2001); Marc Ferro et Jean Planchais, Les médias et l'histoire. Le poids du passé dans le chaos de l'actualité (Paris, CFPJ éditions, 1997).

5. La revue de presse recense les articles de journaux du Québec (quotidiens, hebdomadaires, etc.), les reportages des chaînes de radio et de télévision diffusant dans la capitale nationale, de même que les publicités diffusées dans tous ces médias. Pour les fins de cette analyse, seuls les articles des quotidiens de Québec sont retenus. 
discipline $^{6}$. Le produit présenté par les promoteurs, de même que la couverture médiatique de l'événement, peuvent néanmoins influer sur la perception qu'a le public de l'histoire. Le renouveau de la production historienne sur la Nouvelle-France depuis près de trente ans ${ }^{7}$, notamment en histoire des femmes ${ }^{8}$, constitue par ailleurs une matière à exploiter. Dans ce contexte, il convient de se demander, d'abord, quelle place occupe la diffusion des connaissances historiques dans les objectifs, la programmation, les stratégies de relations publiques et de promotion publicitaire des FNF? Les quotidiens de Québec, de leur côté, se font-ils simples promoteurs ou témoins de la fête, ou profitent-ils de l'occasion pour vulgariser ou parler d'histoire? Enfin, l'image que donnent les médias de la condition féminine en Nouvelle-France répond-elle aux critères de la production historienne actuelle?

\section{L'HISTOIRE AU PROGRAMME}

Les FNF, organisées par Gestev, firme spécialisée en organisation d'événements, ont été lancées en 1997 par la Corporation des fêtes historiques de Québec ${ }^{9}$. En août de chaque année, pendant cinq jours, elles offrent aux nombreux touristes une foule d'activités dans l'arrondissement

6. L'historien Patrice Groulx a mis en lumière les limites de la vulgarisation de l'histoire dans un texte portant sur la production de la télésérie Le Canada, une histoire populaire, «La meilleure histoire du monde», Histoire sociale/Social History, 34 (novembre 2001): 403-414. Au sujet de cette série, voir aussi le texte de Jean-Claude Robert dans ce numéro.

7. Jacques Mathieu, La Nouvelle-France. Les Français en Amérique du Nord, XVI-XVIII siècle (SainteFoy, Les Presses de l'Université Laval, 2001), première édition 1992; Allan Greer, Brève histoire des peuples de la Nouvelle-France (Montréal, Boréal, 1998). La publication en 1974 de l'étude de Louise Dechêne, Habitants et marchands de Montréal au xvIr siècle (Paris, Plon, 1974), en abordant des problématiques dégagées par les approches en histoire sociale, a donné le ton à la production historienne du dernier quart du $\mathrm{xx}^{\mathrm{e}}$ siècle et des premières années du siècle suivant. L'ouvrage collectif, Sylvie Dépatie, Catherine Desbarats, Danielle Gauveau, Mario Lalancette et Thomas Wien, dir., Vingt ans après Habitants et marchands: lectures de l'histoire des XVII et XVIII siècles canadiens (Montréal, McGill-Queen's University Press, 1998), offre un éventail de recherches récentes et d'états des lieux sur la période coloniale. Voir aussi Thomas Wien, "Canada and the Pays d'en haut, 1600-1760", dans Brook Taylor, dir., Canadian History: A Reader's Guide 1: Beginnings to Confederation (Toronto, University of Toronto Press, 1994), 33-75.

8. Les survols historiographiques suivants abordent brièvement la question des femmes en Nouvelle-France : Micheline Dumont, «Un champ bien clos: l'histoire des femmes au Québec», Atlantis, 25,1 (automne 2000): 109-110; Geneviève Ribordy, «La famille en Nouvelle-France: bilan historiographique», Cahiers d'histoire, XII,2 (été 1992): 24-50; Bettina Bradbury, "Femmes et familles ", dans Jacques Rouillard, dir., Guide d'histoire du Québec du Régime français à nos jours. Bibliographie commentée (Québec, Méridien, 1993), 215-217.

9. Le plan directeur adopté en 2000 par la Corporation des fêtes historiques de Québec raconte assez brièvement les origines des FNF. Le rôle joué par l'État n'y est pas abordé, mais sera exploré lors de recherches ultérieures. 
historique du Vieux-Québec: défilés, musique, théâtre, conférences, animations de rue, visites guidées, marché public, concours de costumes, etc. Ces fêtes annuelles dont le succès touristique et économique ne cesse de croître ${ }^{10}$, attirent de nombreux commanditaires d'importance, dont plusieurs médias de la région de Québec. Radio-Canada, Le Soleil et CITF Rock détente ont apporté en 2002 une contribution importante en produits et services ${ }^{11}$, situation créant un potentiel de conflit d'intérêts. Les FNF préparent par ailleurs le terrain pour les célébrations du $400^{\mathrm{e}}$ anniversaire de la fondation de la ville de Québec, en 2008 , déjà objet de débat national ${ }^{12}$. La sixième édition de l'été 2002 retient l'attention en raison des cinq années d'expérience des FNF, qui en font une activité bien rodée, et en raison de la décision des organisateurs d'accorder plus d'importance aux femmes de la colonie, sujet historique méconnu et controversé ${ }^{13}$. Les promoteurs visaient surtout à souligner le rôle des femmes dans la transmission du savoir, thème principal des festivités, mais la condition féminine a été abordée de diverses façons, notamment dans le cadre de conférences scientifiques, de pièces de théâtre et d'animation de rue.

10. L'édition 2001 des Fêtes aurait suscité une augmentation de $30 \%$ de l'achalandage par rapport à l'édition 2000, Régis Tremblay, "La Louisiane aux Fêtes de la Nouvelle-France », Le Soleil, 7 mai 2002. Les organisateurs de l'événement disposaient d'un budget de 2,4 millions de dollars pour l'année 2002, Nicolas Houle, «Des femmes, du savoir et de la... Louisiane », Le Soleil, le 18 juillet 2002.

11. Ces entreprises médiatiques figurent dans la liste des 37 partenaires ayant contribué sous la forme de produits et de services pour une valeur totale de 740500 \$, information fournie dans le rapport d'activités des Fêtes de la Nouvelle-France 2002.

12. Presse canadienne, "Samuel de Champlain au cœur d'un litige», Le Soleil, le 17 août 2001 ; Julie Lemieux, « $400^{\text {e }}$ anniversaire de la fondation de Québec. La capitale n’est pas la seule à vouloir faire la fête à Champlain", Le Soleil, septembre 2001; Raymond Giroux, " $400^{\mathrm{e}}$ anniversaire de la fondation de Québec. Un dossier perdu dans les méandres de la transition à Ottawa », Le Soleil, le 5 septembre 2003.

13. Voir à ce sujet le texte de Jan Noel, «New France: Les femmes favorisées», publié pour la première fois dans la revue Atlantis en 1981, qui a été repris dans plusieurs collections d'essais au cours des années 1980 et jusqu'à la fin des années 1990. Voir notamment Veronica StrongBoag et Anita Clair Fellman, dir., Rethinking Canada. The Promise of Women's History (Toronto, Copp Clark Pitman, 1991), 28-50, deuxième édition. Lire aussi Micheline Dumont, «Les femmes de la Nouvelle-France étaient-elles favorisées? ", Atlantis, 8,1 (1982): 118-124, et la réponse de Jan Noel, 125-130. Lire également la discussion portant sur cette hypothèse d'un âge d'or de la condition féminine en Nouvelle-France dans Josette Brun, «Le veuvage en Nouvelle-France: genre, dynamique familiale et stratégies de survie dans deux villes coloniales du $\mathrm{xvIII}^{\mathrm{e}}$ siècle, Québec et Louisbourg», thèse de doctorat, Université de Montréal, 2000; et Allan Greer, «Les femmes de la Nouvelle-France», dans Brève histoire des peuples de la Nouvelle-France (Montréal, Boréal, 1998), 79-96. 
La diffusion des connaissances historiques est bel et bien au programme des FNF. Le plan directeur précise que les FNF visent à «sensibiliser et développer l'intérêt du public pour l'histoire et la culture». Elles s'adressent "à la population en général, aux familles et à tous ceux que l'histoire intéresse» et doivent se dérouler dans une ambiance propice à «célébrer l'histoire». Leur base scientifique doit par conséquent être solide.

Parce qu'elles évoluent sur le berceau même de la Nouvelle-France, les Fêtes ne peuvent être autrement que crédibles historiquement. Il est donc souhaité et justifié que les Fêtes de la Nouvelle-France trouvent leurs fondements sur un contenu historique valable. Les Fêtes de la Nouvelle-France chercheront à véhiculer avec originalité et sensibilité une histoire à contenu, de différentes $[s i c]$ niveaux, dans le but de mettre en lumière une période historique importante, un univers à découvrir, à comprendre et à partager ${ }^{14}$.

Il ne s'agit pas uniquement de vœux pieux. En 2002, plusieurs conférences scientifiques sont présentées gratuitement au Musée de la Civilisation. Le public peut également consulter, sur le site Internet des Fêtes, les travaux d'étudiants en histoire de l'Université Laval portant sur divers thèmes de l'histoire coloniale. Le "tribunal de l'histoire», activité très courue, fait le procès d'un personnage controversé de la NouvelleFrance en mariant théâtre et connaissance scientifique ${ }^{15}$. Les visites guidées de la ville historique permettent d'en savoir plus long sur la ville et les points d'information historique, et sur l'histoire de la colonie. Des métiers pratiqués à l'époque sont également présentés dans le cadre d'ateliers animés par des artisans ou des bénévoles ${ }^{16}$. L'histoire est également véhiculée de façon moins formelle dans certaines pièces de théâtre et animations de rue, et dans moult autres activités des FNF.

14. Plan directeur adopté par le conseil d'administration de la Corporation des fêtes historiques de Québec, le 29 mars 2000 (désormais: plan directeur).

15. Un personnage sur la sellette répond aux accusations d'une comédienne personnifiant la «Justice» en jouant des textes préparés par un auteur n’ayant pas nécessairement de formation scientifique. Un historien prend par la suite la parole afin de situer le débat ayant cours dans l'historiographie. À la toute fin, le public prend position par un vote.

16. Le programme officiel et le rapport d'activités des FNF 2002 en témoignent. Ce dernier document précise que "Comme à chaque édition, la programmation de l'édition 2002 a été bâtie autour d'un thème permettant un angle de vue sur l'histoire. Cette année, plusieurs animations ont permis d'en apprendre davantage sur "l'éducation en Nouvelle-France, ou la transmission du savoir». Les principales activités figurant au programme des Fêtes y sont énumérées. 
Afin de veiller à la qualité du propos historique, les conseils d'administration et de programmation accueillent depuis les tout débuts un spécialiste de la période coloniale française. Le professeur de l'Université Laval Alain Laberge, avec qui nous avons eu un court entretien, est très sollicité par les médias, qui le citent d'ailleurs à plusieurs reprises. Il joue également un rôle à titre de conseiller en encadrant, autant que faire se peut, le travail de recherche et de création du directeur de la programmation, Jean-François Brochard, et les nombreux bénévoles chargés notamment de l'animation de rue. À ce titre, l'«historien en résidence» des FNF tente surtout de prévenir les dérapages, tout en évitant de jouer les trouble-fête afin de ne pas entraver l'esprit d'innovation et de créativité également à l'honneur ${ }^{17}$. Le directeur de la programmation, monsieur Brochard, affirme dans le même esprit à une journaliste que: «Notre vocation demeure l'histoire, mais l'ambiance festive est tout aussi importante. Nous devons conserver ce côté bon enfant ${ }^{18}$.»

C'est ce dernier aspect des réjouissances qui donne le ton aux stratégies publicitaires et de relations publiques des FNF de 2002. Le plan de communication dit que «[c]ontrairement aux communications des dernières années qui intégraient les thématiques de programmation dans les éléments de communication, cette année le désir est de communiquer d'abord et avant tout l'esprit des Fêtes, à savoir ambiance, atmosphère chaleureuse, convivialité ${ }^{19} »$. On cherche à attirer les visiteurs éventuels en projetant une image de fêtes «excitantes, animées, amusantes» en évacuant leur aspect instructif. Le dépliant officiel des Fêtes reflète bien cette approche puisqu'il montre une photo légèrement stylisée avec en gros plan les pieds dansants d'une femme chaussée de sandales. Seule évocation historique : le tissu de la robe en mouvement qui lui descend jusqu'aux chevilles. Cette image figure sur l'affiche officielle et les publicités publiées dans les médias. Le slogan des FNF de 2002, "Venez faire un saut! », fait également allusion à l'histoire de façon plus implicite qu'explicite. Dans les annonces publicitaires publiées dans les quotidiens de Québec, les grands titres invitent les gens à venir faire un saut "à la fête», "dans nos nouveaux sites» et "en se procurant le programme». Cette dernière publicité précise, en plus petits caractères,

17. Entretien avec Alain Laberge, mai 2003.

18. Katia Bussière, "Une parfaite magie», Journal de Québec, le 12 août 2002.

19. Plan de communication 2002 des Fêtes de la Nouvelle-France. 
que les visiteurs des FNF pourront «faire un saut dans le temps et voyager à travers 400 ans d'histoire ${ }^{20}$ ».

Les raisons ayant mené à l'adoption de cette nouvelle stratégie de communication, de même que le contenu des conférences de presse tenues en 2002, mériteraient d'être explorés en profondeur par le biais d'entrevues avec les promoteurs de l'événement. Il est cependant clair qu'on cherche à accrocher un public de touristes souhaitant d'abord s'amuser en comptant bien sûr sur les médias, souvent à la remorque des activités de relations publiques ${ }^{21}$.

\section{LA COUVERTURE MÉDIATIQUE: L'HISTOIRE AU SECOND RANG}

Les FNF ne manquent pas d'attirer l'attention des médias avant et pendant l'événement qui se tient du 7 au 11 août 2002. Cette couverture médiatique est de toute première importance pour les promoteurs de l'événement qui organisent plusieurs activités de relations publiques à cette fin. Un article publié dans un quotidien peut valoir quelques milliers de dollars en publicité gratuite ${ }^{22}$. Outre les reportages diffusés à la radio et à la télévision, les publicités paraissant dans les journaux, les nouvelles brèves et les publireportages ${ }^{23}$. La revue de presse des Fêtes de 2002 compte 55 articles, dont 20 du journal Le Soleil et 25 du Journal de Québec, qui représentent à eux seuls les quatre cinquièmes de la couverture de la presse écrite québécoise. La proximité géographique de l'événement explique la grande attention que lui portent ces deux journaux, tout comme les enjeux financiers dans le cas du quotidien Le Soleil. Des seize reporters se partageant le travail dans les deux quotidiens de la

20. Publicités des FNF publiées dans Le Soleil, les $1^{\text {er }}$ et 9 août 2002.

21. Au sujet des liens étroits et conflictuels entre professionnels de la communication, consulter Jean Charron, Jacques Lemieux et Florian Sauvageau, Les journalistes, les médias et leurs sources (Boucherville, Gaëtan Morin, 1991) et Armande Saint-Jean, Éthique de l'information: fondements et pratiques au Québec depuis 1960 (Montréal, Presses de l'Université de Montréal, 2002).

22. La valeur estimée varie selon la longueur de l'article, la page où il est publié et le journal qui le met en page. La valeur totale de la revue de presse des FNF 2002 est estimée à deux millions et demi de dollars.

23. Ces publireportages ne sont pas le fait des FNF mais de ses partenaires. Par exemple, l'Université Laval rappelle dans un article que «les Grandes Fêtes de l'Université Laval s'associent aux Fêtes de la Nouvelle-France en présentant un programme aux accents de théâtre et de musique» dans la cour intérieure du Séminaire de Québec, Le Soleil, le 22 juin 2002. De même, la publication $L a$ Terre de chez nous publie des informations de l'Union des producteurs agricoles, qui tient marché sur l'un des sites des FNF, tout comme L'Équipe, journal du personnel de la Société des alcools du Québec, autre commanditaire d'importance. 
capitale, la journaliste du Journal de Québec Katia Bussière est la plus prolifique: elle signe 15 des 25 articles, certains assez courts, publiés par le journal sur les FNF de 2002. Violaine Ballivy écrit pour sa part plusieurs articles à contenu historique pour Le Soleil.

Selon toute attente, l'actualité dame le pion à Clio dans les 45 articles des quotidiens de Québec puisque dominent les "papiers» d'ambiance (14) et les articles à la remorque des activités de relations publiques (10), suivis par ceux liés de plus près aux enjeux de l'heure (problèmes de circulation et programme de commandites du gouver-nement fédéral) ou mentionnant au passage les FNF (chroniques estivales, vente aux enchères d'œuvres d'art, portraits d'artistes invités, fêtes "rivales» de Beauport). Cinq articles font cependant de l'histoire leur sujet principal. Des textes analysés plus loin traitent du rôle joué par Montcalm dans la guerre de la Conquête et du rôle des femmes dans la société coloniale, l'un des thèmes retenus par les organisateurs. Le Soleil publie également un article mettant en lumière les liens historiques entre la NouvelleFrance et la Louisiane ${ }^{24}$, invitée d'honneur des FNF de 2002. Le Journal de Québec se hasarde quant à lui hors des sentiers battus par les Fêtes ${ }^{25}$, pour parler, dans un article intitulé «Nos ancêtres étaient loin d'être des saints", du livre La scandaleuse Nouvelle-France, de Louis Giguère, publié la même année ${ }^{26}$. Répertoire de "faits divers" plutôt qu'analyse scientifique, l'ouvrage fait écho à La vie libertine en Nouvelle-France, publié en 1972 par Robert-Lionel Séguin ${ }^{27}$. Certains propos tenus dans cet article remettent en question les fondements mêmes de la discipline historique. L'auteur affirme en effet à la journaliste que "l'humain ne change pas" et cette dernière renchérit en écrivant: "Autre époque, autre mœurs? Sûrement pas». Elle explique néanmoins que "le code criminel de l'époque, lui, ne ressemblait pas tellement à celui qu'on connaît aujourd'huii ${ }^{28}$ ".

24. Violaine Ballivy, "Nouvelle-France de Mardi gras», Le Soleil, 9 août 2002.

25. Il s'agit selon toute évidence d'une initiative de la journaliste et non de la couverture d'une activité des FNF.

26. Louis Giguère, La scandaleuse Nouvelle-France: histoires scabreuses et peu édifiantes de nos ancêtres (Montréal, Stanké, 2002).

27. Robert-Lionel Séguin, La vie libertine en Nouvelle-France (Ottawa, Leméac, 1972), 2 vol. Au sujet de cette nouvelle vision de la moralité de nos ancêtres qui contrastait de manière flagrante avec celle d'un Lionel Groulx, par exemple, voir Jean Blain, "La moralité en Nouvelle-France: les phases de la thèse et de l'antithèse ", Revue d'histoire de l'Amérique française, 27,3 (décembre 1973): 408-416.

28. Annie Fernandez, «Nos ancêtres étaient loin d'être des saints», Journal de Québec, le 18 juillet 2002 . 
Des journalistes profitent également de la couverture des activités de presse, des articles d'ambiance et des portraits d'artistes pour parler d'histoire ( 11 articles sur 29 , soit le tiers). Le thème qui retient le plus l'attention? La mode en Nouvelle-France! Cette couverture médiatique répond aux objectifs de communication des organisateurs, qui cherchent à nourrir l'ambiance des fêtes en encourageant les gens à se costumer ${ }^{29}$, à instiller chez les gens un souci d'authenticité historique à cet égard, à appuyer les indispensables créateurs de costumes et à attirer les gens à la Place Laurier, centre commercial partenaire des $\mathrm{FNF}^{30}$. C'est là que se tient principalement, en juin, l'exposition itinérante de vêtements style Nouvelle-France "pour tous les goûts, dans tous les styles et pour toutes les bourses ${ }^{31}$ ». L'aspect mercantile de la démarche n'empêche pas toute critique. Le Journal de Québec cite l'organisatrice de l'exposition, Kathy Haton-Brochard, qui affirme que les organisateurs ont «remarqué plusieurs anachronismes pendant les Fêtes de la Nouvelle-France, les gens ayant tendance à confondre cette époque avec l'époque médiévale. Nous croyons important de faire découvrir au grand public les costumes à la mode à l'époque ${ }^{32}$.» La transition entre les Fêtes médiévales et les FNF qui les ont remplacées dans une certaine controverse ne se fait donc pas sans heurts. Le journaliste Richard Boisvert, du journal Le Soleil, écrit cependant que les «robes médiévales aux vastes manches et les culottes bouffantes de la Renaissance se faisaient déjà beaucoup plus rares dans les rues de Québec » en 2001 grâce à l'exposition organisée par les FNF ${ }^{33}$.

Deux mois plus tard, Violaine Ballivy, journaliste au quotidien Le Soleil, fait profiter ses lecteurs des connaissances d'une costumière bénévole, Josée Lebrasseur, qui "s'échine à rétablir certains faits" aux passants, en plein cœur des FNF, sur les rudiments de la fabrication des vêtements. L'intervenante souligne notamment que les «femmes associent la période aux grosses robes, aux broderies et dentelles, alors qu'en

29. Voir le plan de communication 2002 et les publicités des FNF. Dans celle publiée dans Le Soleil du 9 août 2002, les organisateurs lancent l'invitation: «Défilé chaque jour. Costumezvous!».

30. La Place Laurier figure dans la liste de partenaires publiée à la page 12 du rapport d'activités des FNF 2002.

31. Richard Boisvert, "La mode des $\mathrm{xvII}^{\mathrm{e}}$ et $\mathrm{xvIII^{ \textrm {e } }}$ siècles à la portée de tous», Le Soleil, le 4 juin 2002.

32. Marie-France Bornais, "Exposition de costumes - La mode... Nouvelle-France», Journal de Québec, le 4 juin 2002.

33. Richard Boisvert, "La mode des XvII et XviII siècles à la portée de tous", Le Soleil, le 4 juin 2002. 
vérité, la mode à l'époque était toute simple». «Normal, ajoute la journaliste, puisqu'il y avait beaucoup plus de paysans que de nobles et de bourgeois pour défricher le nouveau territoire ${ }^{34}$." L'article décrit par la suite le vêtement des paysannes avant de se pencher sur la question délicate... du port de la culotte! Dans cet esprit légèrement grivois, mentionnons la publicité invitant les gens à assister "aux colorés défilés de costumes d'époque à la scène Place Laurier sur le site des Fêtes de la Nouvelle-France ${ }^{35}$ ». Cette publicité met en scène une femme sagement habillée selon la mode de l'époque mais dont la bretelle retombant négligemment reflète le badin jeu de séduction très présent chez les bénévoles et touristes costumés des FNF. On sent là l'influence des thèses de Robert-Lionel Séguin à propos de l'esprit libertin des colons de la Nouvelle-France. Par ailleurs, ce souci d'authenticité pour le vêtement pourrait refléter une conception de l'histoire centrée sur l'évolution de l'univers matériel.

Le "tribunal de l'histoire», activité présentée par la Commission de la capitale nationale du Québec et reprise dans le cadre des FNF, a attiré l'attention d'une journaliste du quotidien Le Soleil. L'article qu'elle signe au sujet du "procès" théâtral du marquis de Montcalm transmet des informations sur les stratégies militaires durant la guerre de la Conquête. Son texte reprend les principaux arguments avancés par les comédiens jouant le rôle de la "Justice» et de l'officier militaire du $\mathrm{xvIII}^{\mathrm{e}}$ siècle, de même que les propos de l'historien Jacques Lacoursière chargé de faire le point sur la responsabilité de Montcalm dans la défaite de 1760 , avant de se demander si l'on peut vraiment juger en rétrospective... Et de relever l'anachronisme en écrivant que les spectateurs ont "dicté leur sentence en déposant une pierre dans une urne blanche ou noire, selon qu'ils gracient ou non l'accusé, un mode de scrutin jamais employé en Nouvelle-France, mais plutôt en Grèce, pendant $l^{\prime}$ Antiquité ${ }^{36}{ }^{\prime}$. Ce procès marqué par la déconfiture du marquis de Montcalm n'a pas intéressé le Journal de Québec malgré l'aspect conflictuel du débat, qui se prête pourtant bien au récit journalistique ${ }^{37}$. Ce jour-là, la reporter du tabloïd s'est plutôt arrêtée au kiosque de la Fédération des familles-souches québécoises. Elle amorce ainsi son article: "Vous êtes marin, constructeur, notaire ou médecin? Votre ancêtre des années 1600,

34. Violaine Ballivy, «S'éclater costumé pendant 5 jours», Le Soleil, le 8 août 2002.

35. "La mode de tout temps» (publicité), Journal de Québec, le 10 août 2002.

36. Violaine Ballivy, « Montcalm - coupable!», Le Soleil, le 10 août 2002.

37. Pierre Sormany, Le métier de journaliste (Montréal, Boréal, 2000). 
nouvellement arrivé en Nouvelle-France, exerçait probablement la même profession. C'est fascinant de voir à quel point le même métier se transmet dans une même lignée de générations. Fruit du hasard ou gène commun? Personne ne le $\operatorname{sait}^{38}$.» La journaliste ajoute que la porteparole de la Fédération trouve "curieux [que] les goûts reviennent ainsi de génération en génération». Nulle mention du phénomène de la reproduction sociale, bien connu en histoire, qui cède ici la place au déterminisme biologique et à la chance.

\section{UN CHAMP MÉCONNU: L'HISTOIRE DES FEMMES}

Les critiques adressées aux organisateurs des FNF sur la place effacée des femmes, de même que le débat historiographique entourant leur rôle dans la société coloniale, ont trouvé peu d'écho dans les quotidiens de Québec en 2002. L'intérêt pour la controverse est pourtant caractéristique des pratiques journalistiques actuelles ${ }^{39}$. C'est une reporter de La Presse, à Montréal, qui rappelle que

[1]'an dernier, à pareille date, on reprochait aux Fêtes de la Nouvelle-France de ne pas accorder assez d'importance aux personnages féminins de l'époque. Cette année, l'organisation fait amende honorable. Le rôle déterminant des femmes aux premières heures de la colonie et leur apport à la transmission du savoir sont au cœur des Fêtes cuvée 2002, qui étaient lancées mercredi, dans la Vieille Capitale ${ }^{40}$.

Le Journal de Québec mentionne plus discrètement que «[1]'organisation des Fêtes de la Nouvelle-France a voulu, cette année, mettre en évidence le savoir-faire de ces femmes à travers des activités d'animation historiques et culturelles ${ }^{41}$ ». L'occasion de discuter de l'histoire des femmes comme spécialisation relativement nouvelle et peu connue du public n'a pas été saisie au vol.

La condition des femmes en Nouvelle-France intéresse par ailleurs les reporters beaucoup plus que les personnages féminins bien connus se prêtant pourtant bien au récit journalistique ${ }^{42}$. Ce choix correspond tout

38. Katia Bussière, "Un passé surprenant», Journal de Québec, le 10 août 2002.

39. Sur les médias, les pratiques journalistiques et les questions féministes, voir Barbara N. M. Freeman, The Satellite Sex. The Media and the Women's Issues in English Canada, 1966-1971 (Waterloo, Wilfrid Laurier University Press, 2001), ainsi que Micheline Dumont et Stéphanie Lanthier, «Pas d'histoire, les femmes! Le féminisme dans un magazine québécois à grand tirage : L'actualité 1960-1996», Recherches féministes, 11,2 (1998): 101-124.

40. Ève Dumas, "Les Fêtes des femmes», La Presse, le 9 août 2002.

41. Katia Bussière, "Le savoir-faire des femmes de la colonie», Journal de Québec, le 10 août 2002.

42. Barbara Freeman, The Satellite Sex..., op. cit. 
à fait aux critères de la production scientifique actuelle ${ }^{43}$. La figure officielle des Fêtes (et de l'effigie donnant accès aux sites), Marie Rollet, pionnière et épouse de Louis Hébert, passe pratiquement inaperçue ${ }^{44}$. La fondatrice des Ursulines de Québec, Marie de l'Incarnation, est également absente du discours médiatique, malgré le rôle de tout premier plan qu'elle a joué dans la colonie et malgré la conférence scientifique prononcée par Claire Gourdeau sur les éducatrices dans le cadre des $\mathrm{FNF}^{45}$. Ainsi, plutôt que de traiter de cette célèbre fondatrice et missionnaire, une journaliste du Journal de Québec s'intéresse, de façon générale, à l'éducation des femmes et à leur rôle dans la colonie, thème d'ailleurs privilégié par les promoteurs des Fêtes. Elle se demande ce que serait devenue la Nouvelle-France sans tout le savoir des femmes:

Car les hommes, partis au loin dans le bois, ne s'occupaient guère de la maison. Ce sont les femmes qui tenaient la demeure, éduquaient les enfants, faisaient les comptes, veillaient aux cultures du sol et soignaient le bétail. Leur savoir, les femmes l'apprenaient sur les bancs d'école, chez les ursulines par exemple. «Les religieuses enseignaient aux petites filles bourgeoises, amérindiennes ou françaises comment devenir une bonne épouse et une bonne mère au foyer ", affirme Catherine Seres, directrice adjointe du Musée des ursulines. Les fillettes apprenaient à lire, à écrire et à jeter (compter avec les jetons), mais aussi les techniques manuelles telles que la broderie. L'école durait entre six semaines et huit mois. "Être éduquée, ce n’était pas que la pédagogie ou les matières scolaires, c'était également la transmission des bonnes manières et de la morale, selon son statut social", soutient l'historien Alain Laberge ${ }^{46}$.

Le Soleil se penche pour sa part sur le statut des femmes en NouvelleFrance. L'article reprend l'interprétation selon laquelle les Canadiennes auraient bénéficié d'un statut élevé et d'un champ d'action élargi par rapport à leurs contemporaines françaises. Le titre est sans équivoque :

43. Larue et Montal rappellent que l'«évocation et la production des héros est un genre dépassé de la connaissance historique». Il faut, disent-ils, "dénoncer, de manière collective, la reprise de ces grands récits dans une optique politique et commerciale», "Québec 1984», loc. cit., 55. Sur la question des héroïnes, voir aussi Colin M. Coates et Cecilia Morgan, Heroines and History: Representations of Madeleine de Verchères and Laura Secord (Toronto, University of Toronto Press, 2001).

44. Sur les pionnières, voir le Collectif Clio, L'histoire des femmes au Québec depuis quatre siècles (Québec, Le Jour, 1992), 19-148.

45. Claire Gourdeau est l'auteure de l'ouvrage Les délices de nos coeurs: Marie de l'Incarnation et ses pensionnaires amérindiennes, 1639-1672 (Sillery, Septentrion, 1994).

46. Katia Bussière, "Le savoir-faire des femmes de la colonie», Journal de Québec, le 10 août 2002. 
«Terre de liberté des femmes. Leur rôle était plus important ici qu’en France.» Cette conception des choses est appuyée par l'historien Jacques Lacoursière qui raconte qu'en Nouvelle-France circulait le dicton suivant: "cinq mille pieds passé le village, un homme n’est plus marié », avant d'expliquer que ces quelques mots

n'évoquent pas tant la faiblesse de la chair des premiers colons devant les charmes des belles autochtones que la liberté de leurs épouses lorsqu'ils partaient en mission, perdus dans l'immensité du territoire. Guerres, voyages d'exploration, commerce, les raisons étaient nombreuses pour les hommes de s'éloigner de la maison, de longs mois durant, pendant lesquels les femmes devaient se débrouiller, fin seules. Prenant ni plus ni moins la relève de leur mari, elles s'occupaient de tout, de l'éducation des enfants comme des finances du foyer. "Avant de partir, les hommes signaient des procurations à leurs épouses, leur donnant le contrôle du logis. En fait, elles avaient beaucoup plus de liberté ici qu'en France», avance l'historien. À la même époque, sur le Vieux Continent, elles n'auraient sans doute jamais eu de telles responsabilités ${ }^{47}$.

Dans le même article, la journaliste affirme que "[s]i les premières arrivées ont pu bénéficier d'autant de considération, c'est sans doute aussi grâce aux échanges avec les tribus autochtones ». À l'appui de cette thèse, elle cite l'anthropologue et métisse Nathalie Saint-Pierre, conférencière sur les femmes autochtones dans le cadre des FNF, qui explique que «dans les nations amérindiennes $[. .$.$] la femme n'avait pas un rôle$ plus important que celui de l'homme, mais il était équivalent et très respecté ${ }^{48}$ ». La journaliste nuance cependant son propos dans la conclusion de son texte où elle affirme que les Blanches avaient un statut moins avantageux que les femmes autochtones: «[d]u côté colonial, les femmes n'ont jamais eu ce privilège [d'être les égales des hommes], du moins pas de façon aussi ouverte. Malgré tout, elles pouvaient à tout le moins se consoler en pensant qu'elles avaient plus d'emprise sur la vie quotidienne des hommes ici que n'en avaient leurs compatriotes restées outre-mer ${ }^{49}$.»

Le débat historiographique sur le statut et le rôle des femmes dans la société coloniale française des $\mathrm{xVII}^{\mathrm{e}}$ et $\mathrm{xVIII}^{\mathrm{e}}$ siècles est absent de la

47. Violaine Ballivy, "Terre de liberté des femmes. Leur rôle était plus important ici qu’en France ", Le Soleil, le 11 août 2002.

48. Ibid.

49. Ibid. 
couverture journalistique ${ }^{50}$. Une conception positiviste de l'histoire dispenserait-elle les reporters de présenter «les deux côtés de la médaille", règle d'or du journalisme, quand il s'agit d'un sujet à caractère historique? Il semblerait, en effet, que dans ces circonstances, la version présentée par un expert est considérée comme la seule version possible. Quoi qu'il en soit, l'écart entre l'état des connaissances et le discours des médias à cet égard met en lumière les limites de la démarche de vulgarisation. La lente et difficile intégration de l'histoire des femmes dans le discours scientifique relayé par les historiens porte également à conséquence ${ }^{51}$. Malgré l'intérêt des journalistes pour une histoire plus sociale qu'héroïsante, les contraintes du métier et les valeurs qui encadrent sa pratique rendent difficile la couverture en profondeur de questions complexes ${ }^{52}$. Elles permettent néanmoins une couverture originale et vivante, souvent même instructive malgré quelques dérapages.

Quant aux historiens, ils se font plutôt silencieux lors de la tenue d'événements du genre, sauf en tant que porte-parole ou participants. Les historiens extérieurs aux Fêtes, s'ils n’ont pas été sollicités par les médias comme figures d'autorité non officielles, ne se sont pas plus manifestés dans les rubriques d'opinion du lecteur ou dans les pages d'idées des quotidiens. La revue de presse ne recense en effet aucune appréciation des FNF 2002 de la part de spécialistes du domaine ${ }^{53}$. Cette

50. Un autre courant d'interprétation souligne plutôt les ressemblances avec les sociétés française, anglo-américaine et canadienne-française : différenciation et hiérarchisation des sexes, autorité maritale et incapacité juridique des femmes mariées, crainte de la sexualité et du pouvoir féminins, poids du vieillissement, des conventions sociales et du discours religieux chez les femmes. Outre les textes mentionnés plus haut, voir France Parent et Geneviève Postolec, "Quand Thémis rencontre Clio : les femmes et le droit en Nouvelle-France », Les Cahiers de Droit, 36,1 (mars 1995) : 293-318; Marie-Aimée Cliche, «Filles-mères, familles et société sous le Régime français", Histoire sociale/Social History, 21,41 (mai 1988): 39-70; Maurice Basque, "Genre et gestion du pouvoir communautaire à Annapolis Royal au $18^{\mathrm{e}}$ siècle », Dalhousie Law Journal, 17,2 (automne 1994): 498-508; et Peter Moogk, "Thieving Buggers and Stupid Sluts: Insults and Popular Culture in New France", William and Mary Quarterly, 36,4 (octobre 1979) : 524-547.

51. Micheline Dumont, Découvrir la mémoire des femmes: une historienne face à l'histoire des femmes (Montréal, Éditions du remue-ménage, 2001); Andrée Lévesque, "Réflexions sur l'histoire des femmes dans l'histoire du Québec", Revue d'histoire de l'Amérique française, 51,2 (automne 1997): 271-284.

52. Au sujet de l'apparition de pratiques journalistiques axées moins sur l'information que la communication, voir Jean Charron et Jean de Bonville, "Le paradigme du journalisme de communication: essai de définition", Communication, 17,2 (1996): 51-97.

53. La revue de presse devait-elle en faire état? Un dépouillement des rubriques d'opinion du lecteur des quotidiens de Québec permettrait de vérifier si des lettres ont été publiées à ce sujet. 
absence de critiques, qui mériterait un examen approfondi, a déjà été observée, en 1984, lors du $450^{\mathrm{e}}$ anniversaire de l'arrivée de l'explorateur Jacques Cartier ${ }^{54}$. L'aspect commercial de ce type de célébration empêcherait par ailleurs, selon ces mêmes auteurs, la constitution de l'histoire comme science critique. Des commentaires provenant d'autres sources remettent en question, dans une autre optique, la logique marchande au cœur de ces fêtes récréotouristiques. Ainsi, on cite, dans Le Soleil, l'organisateur des Fêtes au bourg, qui ont lieu chaque année depuis l'an 2000 dans le quartier historique de Beauport, qui affirme que «[c]e n'est pas du tout la même ambiance que les Fêtes de la Nouvelle-France, qui sont devenues très commerciales à mon avis. Ici, c'est plus paysan, plus collé sur l'histoire locale, plus proche des gens ${ }^{55}$.» Le même journal, commanditaire des FNF, cite également Laurence, une jeune femme de 19 ans, qui «regrette que les articles mis aux enchères [lors des encans ayant lieu dans le cadre des Fêtes] ne soient pas tous reliés à la vie en Nouvelle-France. Vendredi, on proposait, entre autres, des bons valables dans des restaurants, des disques, un assortiment de bières... "Je m'attendais à autre chose, à ne voir que des éléments qui représentent la vie en Nouvelle-France. Mais les commanditaires veulent faire de la publicité en offrant leurs produits", estime-t-elle ${ }^{56}$.»

Ces rapports complexes entre l'histoire, le commerce et la communication de masse seront étudiés plus avant dans le cadre d'une étude couvrant les FNF depuis leurs débuts en 1997. Une telle recherche permettra notamment de situer l'évolution des stratégies de communication publique des organisateurs et d'établir la représentativité de la revue de presse de l'année 2002. Les modalités de la couverture radiophonique et télévisuelle restent à découvrir, tout comme la différence du traitement de l'information dans les médias francophones et anglophones du Québec et du Canada. Le rôle et l'influence des partenaires et commanditaires des fêtes commémoratives mériteront également notre attention. L'analyse des sondages commandés par les promoteurs des FNF donnera par ailleurs une autre mesure de l'intérêt qu'ils portent, ainsi que les visiteurs, à la transmission du savoir, et de l'effet du contenu des célébrations sur le public. Histoire vivante à suivre...

54. Richard Larue et Fabrice Montal, "Québec 1984», loc. cit., 50.

55. Mylène Moisan, "Les Fêtes au bourg", Le Soleil, le 5 août 2002.

56. Auteur non identifié, «La monnaie de carte se dépense à la vente aux enchères », Le Soleil, le 11 août 2002. 